\title{
Sequential Design Process for Screening and Optimization of Robustness and Reliability Based on Finite Element Analysis and Meta-Modeling
}

Brix Nerenst, Tim; Ebro, Martin; Nielsen, Morten; Bhadani, Kanishk; Asbjörnsson, Gauti; Eifler, Tobias; Nielsen, Kim L.

Published in:

Journal of Computing and Information Science in Engineering

Link to article, DOI:

$10.1115 / 1.4053074$

Publication date:

2022

Document Version

Peer reviewed version

Link back to DTU Orbit

Citation (APA):

Brix Nerenst, T., Ebro, M., Nielsen, M., Bhadani, K., Asbjörnsson, G., Eifler, T., \& Nielsen, K. L. (2022).

Sequential Design Process for Screening and Optimization of Robustness and Reliability Based on Finite Element Analysis and Meta-Modeling. Journal of Computing and Information Science in Engineering, 22(4), [040902]. https://doi.org/10.1115/1.4053074

\section{General rights}

Copyright and moral rights for the publications made accessible in the public portal are retained by the authors and/or other copyright owners and it is a condition of accessing publications that users recognise and abide by the legal requirements associated with these rights.

- Users may download and print one copy of any publication from the public portal for the purpose of private study or research.

- You may not further distribute the material or use it for any profit-making activity or commercial gain

- You may freely distribute the URL identifying the publication in the public portal 


\section{SEQUENTIAL DESIGN PROCESS FOR SCREENING AND OPTIMIZATION OF ROBUSTNESS AND RELIABILITY BASED ON FINITE ELEMENT ANALYSIS AND META-MODELING}

\author{
Tim Brix Nerenst* \\ Section of Solid Mechanics \\ Dep. of Mechanical Engineering \\ Technical University of Denmark \\ Kgs. Lyngby, 2800, Denmark \\ Email:TBNE@MEK.DTU.DK

\section{Kanishk Bhadani \\ Gauti Asbjörnsson} \\ Dep. of Industrial and Materials Science \\ Chalmers University of Technology \\ SE-412 96 Göteborg \\ Sweden
}

\author{
Martin Ebro \\ Morten Nielsen \\ Modelling \& Simulation \\ Novo Nordisk A/S \\ Hilleroed, 3400 \\ Denmark \\ Tobias Eifler \\ Kim Lau Nielsen \\ Dep. of Mechanical Engineering \\ Technical University of Denmark \\ Kgs. Lyngby, 2800 \\ Denmark
}

\section{ABSTRACT}

A new medical device can take years to develop from early concept to product launch. The long development process can be attributed to the severe consequences for the patient if the device malfunctions. As a result, three approaches are often combined to mitigate risks: Failure Modes and Effects Analysis (FMEA), simulation and modeling, and physical test programs. Although widely used, all three approaches are generally time-consuming and have their shortcomings: The risk probabilities in FMEA's are often based on educated guesses, even in later development stages as data on the distribution of performance is not available. Physical test programs are often carried out on prototype components from the same batch and, therefore, may not reveal the actual distribution of actual running performance. Finally, simulation and modeling are usually performed on nominal geometry - not accounting for variation - and only provide a safety factor against failure. Thus, the traditional use of safety factors in structural analysis versus the probabilistic approach to risk management presents an obvious misfit. Therefore, the above three approaches are not ideal for addressing the design engineer's key question; how should the design be changed to improve robustness and failure rates. The present work builds upon the existing Robust and Reliability-Based Design Optimization $\left(R^{2} B D O\right)$ and adjusts it to address the key questions above using Finite Element Analysis (FEA). The two main features of the presented framework are screening feasible design concepts early in the embodiment phase and subsequently optimizing the design's probabilistic performance (i.e., reduce failure rates) while using minimal computational resources. A case study in collaboration with a medical design and manufacturing company demonstrates the new framework. The case study includes FEA contact modeling between two plastic molded components with twelve geometrical variables and optimization based on meta-modeling. The optimization minimizes the failure rate (and improves design robustness) concerning three constraint functions (torque, strain, and contact pressure). Furthermore, the study finds that the new framework significantly improves the component's performance function (failure rate) with limited computational resources.

Keywords: Reliability, Robustness, DOE, Optimization, FEA. 


\section{INTRODUCTION}

Robustness and reliability evaluations are essential in the field of medical injection devices development due to the severity of consequences for the patient, especially in the cases of malfunctions and misuses. A common practice of designing and developing medical products is based on adding safety factors to the expected nominal performance. The safety factor ensures that a given failure threshold accounts for the inevitable geometrical variation and depends on the selected material and manufacturing method. However, the application of safety factor practice has inherent drawbacks as it requires empirical data to determine the target performance values without either over-engineering or over-looking a risk. Furthermore, a safety factor against a failure mode is challenging to translate into a probability of failure, which is needed for risk management, e.g., an FMEA. As an alternative design theory, probabilistic approaches aimed to estimate the risk based on statistical input have been utilized for development [1]. Modern approaches such as Robust Design Optimization (RDO) and Reliability-Based Design Optimization (RBDO), established by the automotive, aerospace, and defense industries, have been developed to reduce safety factors' inefficiencies [2,3]. Robust Design Optimization (RDO) aims to reduce the impact of variation on the targeted performance mean, i.e., minimize the design sensitivity, often measured by the size of the standard deviation [4-6]. In contrast, Reliability-Based Design Optimization (RBDO) aims to reduce the probability of failure, i.e., minimize the failure rate by optimizing the ideal distance to a failure constraint [7]. The researchers in [8-10] have further advocated for combining RDO and RBDO approaches, which have resulted in Robust and Reliability-Based Design Optimization $\left(R^{2} B D O\right)$ [11]. In brief, $R^{2} B D O$ is an optimization technique to identify designs less sensitive to variation while simultaneously minimizing the probability of failure. As these requirements are often in conflict, the $R^{2} B D O$ attempts to satisfy both requirements to avoids sub-optimal designs. The $R^{2} B D O$ approach has been implemented successfully for industrial product design where legacy knowledge is available and incremental improvements are essential [11]. However, the conventional $R^{2} B D O$ approach is unsuitable for medical devices tailored for new market opportunities. For example, a device could require a new activation mechanism, an alternative delivery system, or specific drug requirements. As a result, the development engineers face new design challenges and limited legacy knowledge (missing models, unknown failure modes). While the engineers' end goal is to develop a robust and reliable device, gaining fundamental design understanding is vital, wherein iterations and concept changes are inevitable. Additionally, multiple competing design concepts are often developed in parallel to increase the chance of success $[12,13]$. However, $R^{2} B D O$ is computationally expensive due to the complexity of the Finite Element Analysis (FEA) and the Design Of Experiment (DOE)/meta-modeling requirements. Therefore, the approach is computationally imprac- tical to apply for multiple design concepts with multiple load cases. Consequently, the application of $R^{2} B D O$, in its current form, is limited when developing a new medical device.

The present work demonstrates a sequential Robust and Reliability-based Design Optimization framework $\left(s R^{2} B D O\right)$ tailored to design and develop multiple competing design concepts with limited legacy knowledge. To demonstrate the framework's applicability, a case study pertaining to specific medical device components is presented. The significant advantage of this application is extracting design knowledge through the multiobjective optimization process while minimizing the computational expenses using augmented DOE and meta-models.

The paper is structured as follows; Section 2 outlines the new $s R^{2} B D O$ using a case study example. In section 3 the obtained results of the framework and case study are presented. Section 4 outlines the discussion of potential limitations and industrial application. Finally, section 5 summarizes the main conclusions of the study.

\section{METHOD AND CASE STUDY}

The proposed $s R^{2} B D O$ framework is illustrated in Fig. 1. The framework separates the analysis into a "Design insight" block and a "Design optimization" block. The purpose of separating the $s R^{2} B D O$ framework into two sequential blocks are i) an efficient screening (variables, performance) of new design concepts in the early embodiment phase and ii) a targeted use of computational resources focused on the more promising design concepts. In practice, the complexity and the computational expense of simulations (here based on finite element analysis) typically prevent direct optimization, and meta-models are therefore required. Latin Hypercube Sampling combined with Kriging is often preferred to generate accurate meta-models for non-linear systems [14]. However, evaluating multiple design concepts and load cases becomes computationally expensive by adopting such approaches. Instead, a smaller experimental design is conducted in block 1 in the $s R^{2} B D O$ to initially screen the design concepts for a feasible solution space before the subsequent optimization. To "screen" is defined as evaluating variable sensitivities and the ratio of constrained and non-constrained designs with respect to performance requirements. After feasible designs are identified, augmentation of the existing DOE is performed in block 2 to increase the meta-models' accuracy and making them useful for optimization. This sequential approach yields a significant reduction in the number of simulations required by disregarding design concepts without potential using block 1. Moreover, a further reduction in simulation expenses is achieved by only augmenting the DOE for the statistically significant variables identified from block 1. A cost example is shown in Figs. 2 and 3. Considering the case parameters of multiple competing design concepts, multiple load cases, and variables, the cost of performing $s R^{2} B D O$ would, for this case, be almost three times as inex- 


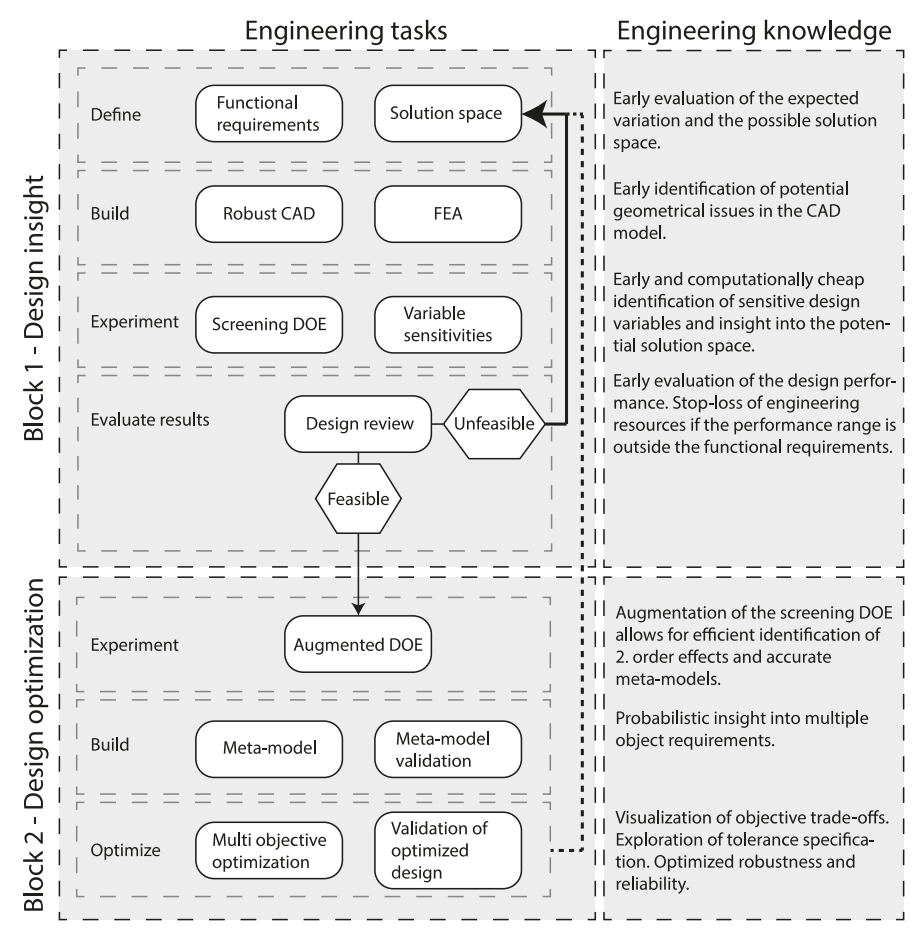

FIGURE 1. THE NEW SEQUENTIAL $R^{2} B D O$ FRAMEWORK.

pensive.

The following sections detail the individual steps in the framework while considering a case study. The description includes one design concept and load case to showcase the steps of the two blocks in Fig. 1. To evaluate the efficiency and accuracy of the new sequential framework, a direct comparison against the original $R^{2} B D O$ process using an Optimal Latin Hypercube with 120 new simulations is made at the end of the result section.

\section{Block 1 - Functional Requirements and Solution Space}

The initial step is to set the task objectives by defining the functional requirements, the design variables, and the allowable solution space for the variables [13]. Figure 4 shows a part of an insulin injection device concept that consists of two components. Figure 5 shows a graphical representation of the twelve geometrical variables (red) and the three functional requirements (blue) on component A. The functional requirements are specified for a minimum torque and maximum strain and contact pressure. The strain and contact pressure locations were identified as problematic during the initial nominal FEA. A feasible optimization solution requires; i) the torque must be higher than $90 \mathrm{Nmm}$ to ensure the relative rotational locking after assembly adjustment. ii) The average strain between the teeth on component $\mathrm{A}$ must be less than 0.024 due to a weld line from the molding process. iii) The average contact pressure on the teeth must be less than $75 \mathrm{MPa}$ to avoid scraping off a lubricating coating. Creating a visual representation of the geometrical variables and require-
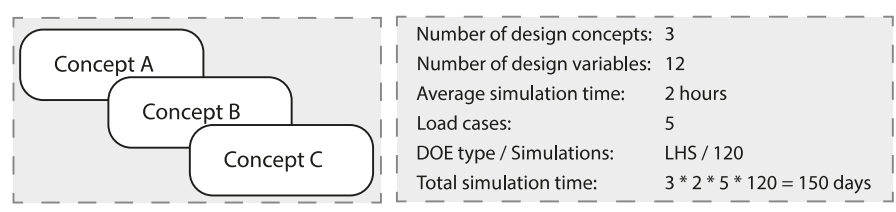

FIGURE 2. THE ESTIMATED COMPUTATIONAL COST FOR THE ORIGINAL $R^{2} B D O$ APPROACH. THE EXAMPLE CONTAINS THREE DESIGN CONCEPTS, EACH WITH FIVE LOAD CASES AND TWELVE VARIABLES.

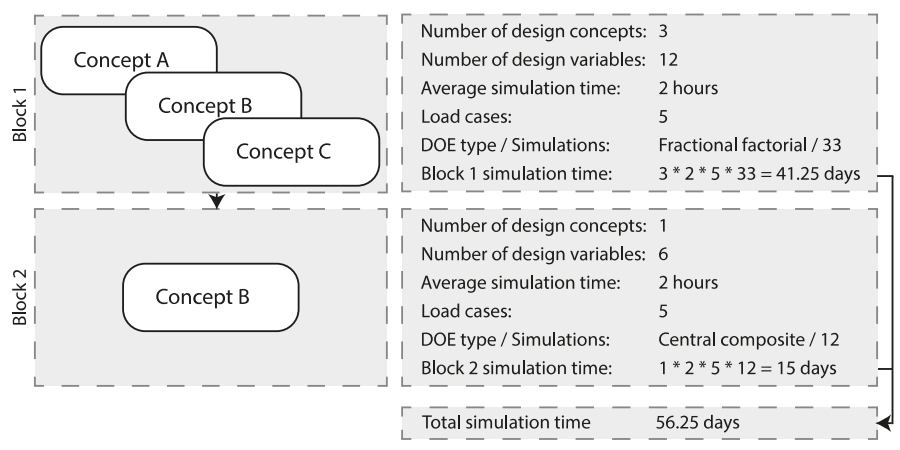

FIGURE 3. THE ESTIMATD COMPUTATIONAL COST FOR THE SUGGESTED $s R^{2} B D O$ FRAMEWORK. THE EXAMPLE CONTAINS THREE DESIGN CONCEPTS, EACH WITH FIVE LOAD CASES AND TWELVE VARIABLES.

ments, as shown in Fig. 5, is considered a key deliverable in block 1 of the new $s R^{2} B D O$ framework. A visual representation is an efficient communication method and ensures all engineers agree on the exact dimensions and locations of change. It is recommended to have a 1-to-1 alignment between the graphical representation and the definition of variables in the Computer Aided Design (CAD) model. For example, in Fig. 5, the thicknesses (T) is a direct dimension instead of a function of an inner and outer radius. This step is linked directly to the validity of the CAD model, which must ensure all the geometrical combinations can be generated (to ensure successful FEA simulations). Table 1 shows the variable definitions. For the optimization, maximum allowable geometrical changes were set to $\pm 2.5 \%$ for all variables, referred to as the Solution Space Variation (SSV). SSV controls the geometrical limits to develop feasible solutions. The specification here is dependent on the design engineering knowledge about the particular product and production method. In this case study, a limited solution space of $\pm 2.5 \%$ is selected to avoid geometrical conflicts with other close-by components. An additional uncertainty variation is required to estimate the robustness and reliability. Robustness is defined as a measure of the standard deviation of performance from its mean [15]. Reliability is defined as the probability of feasible designs, i.e., the inverse of the failure rate [15]. In this case, the Estimated Uncertainty Variation (EUV) represents tolerances of $\pm 1.0 \%$. EUV represents the inherent uncontrolled variation in the geometric design variable 


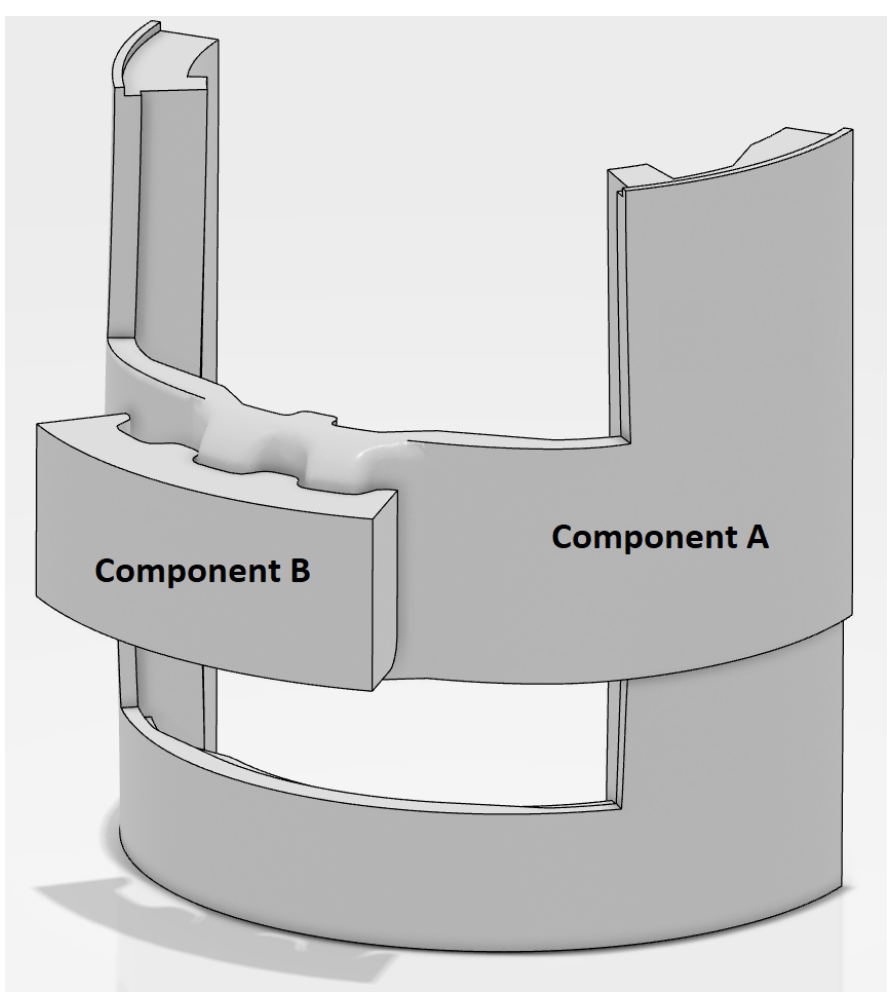

FIGURE 4. THE TWO COMPONENTS INVESTIGATED IN THE CASE STUDY. THE COMPONENTS ARE A SUBSET OF THE INTERNAL COMPONENTS OF AN INJECTION DEVICE. THE TWO COMPONENTS ARE CYLINDRICAL BUT SECTIONED TO REDUCE THE NUMBER OF ELEMENTS.

due to the selected manufacturing process, material, and product use over time. The specification is dependent on the engineering knowledge, such as the allowed tolerance associated with a particular product. The $\pm 1.0 \%$ is selected in this case as it reflects the standard process variation of an IT-grade 13, a commonly used tolerance specification for large-scale production of plastic molded parts [16].

\section{Block 1 - Robust CAD and FEA}

The efficiency of the $R^{2} B D O$ process depends on the size of the experimental design. For example, a fractional factorial design with resolution III can be a good candidate for the screening. Resolution III allows for a compact design for the initial identification of main effects and some interactions. However, the drawback of reduced experimental designs with only a few sampling points is its sensitivity to data loss. Therefore, an effort must be made to ensure that all geometrical combinations can be generated within the specified SSV and simulated without errors. Ensuring a flawless simulation process, therefore, require focus on robust $\mathrm{CAD}$ and FEA, which separates this framework from others alike [17].

In this study, the CAD components are created with the Re-

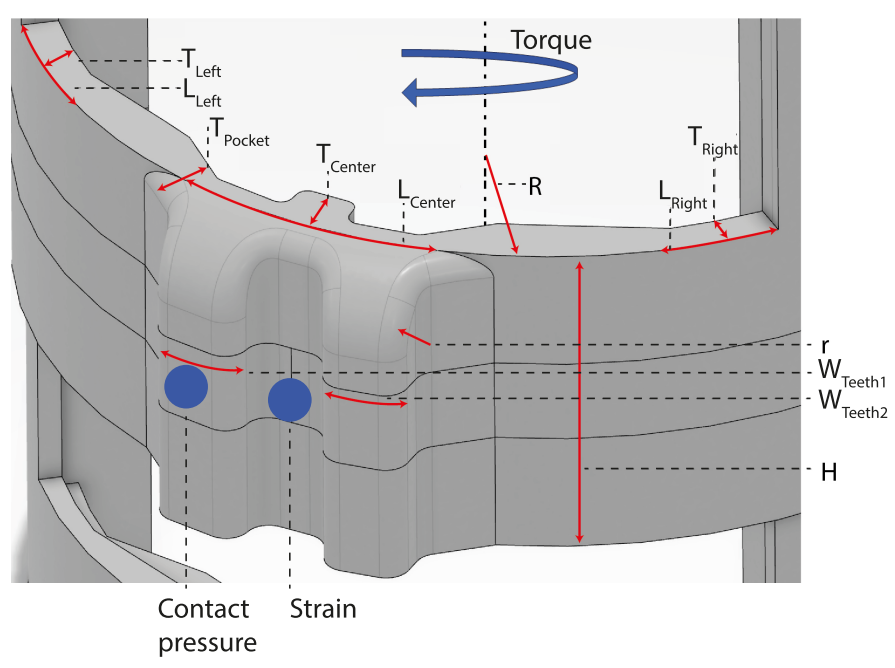

FIGURE 5. VISUAL REPRESENTATION OF THE SELECTED GEOMETRICAL VARIABLES (RED) AND THE FUNCTIONAL REQUIREMENTS (BLUE) INVESTIGATED FOR COMPONENT A (SEE FIG. 4). THE VISUALIZATION IS SUITABLE TO COMMUNICATE THE GOAL OF THE STUDY.

silient Modeling Strategy [18] in order to increase the CAD robustness [19]. The strategy follows a strict order of features to minimize the non-robust relations between parent and child features when regenerating the CAD model with a new parameter set. Secondly, dynamic partitioning was created for the strain and contact pressure zones to ensure an accurate comparison of results for each simulation. Successful regeneration of the CAD enables fully automating the DOE-FEA process, as the mesh model can regenerate automatically - minimizing the need for human interaction, errors, and long process time. The FEA setup is presented as an overview in Table 2. Two steps are required to ensure the FEA's robustness, a positioning step (translation) and a result step (rotation). The positioning step is required as some geometrical combinations might cause initial overlap between the components, otherwise terminating the simulation. The simulation time per FEA is one hour on a DELL workstation (16x Intel(R) Xeon(R) CPU E5-2690 v4 @ 2.60GHz).

\section{Block 1 - Screening DOE and Sensitivity}

The framework requires a computationally economical strategy for generating initial data for screening, and so useful experimental design candidates are Fractional Factorial III, PlackettBurman, and Definitive Screening Designs (DSD). PlackettBurman is primarily efficient if the variables are a multiple of four with a starting point of eighth. Definitive Screening is an alternative that prevents confounding of factors and can also detect non-linear responses. In initial attempts, it is recommended to apply a fractional factorial design due to its matrix simplicity, the flexibility of the number of included variables, and standard- 
TABLE 1. DEFINITION OF THE GEOMETRICAL VARIABLES. THE OVERVIEW CONTAINS THE ORIGINAL NOMINAL VALUES, THE SOLUTION SPACE VARIATION (SSV), THE EXPECTED UNCONTROLLED VARIATION (EUV), AND THE DISTRIBUTIONS OF THE VARIATION.

\begin{tabular}{|c|c|c|c|c|c|c|}
\hline Var. & Var. & Norm. & SSV & EUV & EUV & EUV \\
\hline Name & Sym. & Value & Range & Sym. & Range & Distr. \\
\hline$[-]$ & {$\left[x_{i}\right]$} & {$[\mathrm{mm}]$} & {$[\%]$} & {$\left[\sigma_{i}\right]$} & {$[\%]$} & {$[-]$} \\
\hline$W_{\text {Teeth } 1}$ & $x_{1}$ & 0.778 & \pm 2.5 & $\sigma_{1}$ & \pm 1.0 & norm \\
\hline$W_{\text {Teeth2 }}$ & $x_{2}$ & 0.628 & \pm 2.5 & $\sigma_{2}$ & \pm 1.0 & norm \\
\hline$T_{\text {Pocket }}$ & $x_{3}$ & 0.436 & \pm 2.5 & $\sigma_{3}$ & \pm 1.0 & norm \\
\hline$T_{\text {Right }}$ & $x_{4}$ & 0.300 & \pm 2.5 & $\sigma_{4}$ & \pm 1.0 & norm \\
\hline$T_{L e f t}$ & $x_{5}$ & 0.300 & \pm 2.5 & $\sigma_{5}$ & \pm 1.0 & norm \\
\hline$T_{\text {Center }}$ & $x_{6}$ & 0.460 & \pm 2.5 & $\sigma_{6}$ & \pm 1.0 & norm \\
\hline $\mathrm{R}$ & $x_{7}$ & 4.950 & \pm 2.5 & $\sigma_{7}$ & \pm 1.0 & norm \\
\hline $\mathrm{H}$ & $x_{8}$ & 2.724 & \pm 2.5 & $\sigma_{8}$ & \pm 1.0 & norm \\
\hline$L_{L e f t}$ & $x_{9}$ & 0.963 & \pm 2.5 & $\sigma_{9}$ & \pm 1.0 & norm \\
\hline$L_{\text {Right }}$ & $x_{10}$ & 0.925 & \pm 2.5 & $\sigma_{10}$ & \pm 1.0 & norm \\
\hline$L_{\text {Center }}$ & $x_{11}$ & 3.345 & \pm 2.5 & $\sigma_{11}$ & \pm 1.0 & norm \\
\hline $\mathrm{r}$ & $x_{12}$ & 0.200 & \pm 2.5 & $\sigma_{12}$ & \pm 1.0 & norm \\
\hline
\end{tabular}

ized post-processing.

A 2-level fractional factorial design is selected in this case study with resolution IV for computational and simplicity reasons. The design and resolution are based on expected interaction terms and the assumption of the effect sparsity principle [20], stating that most likely, only a limited number of main variables and lower order interactions are statistically significant. The 2-levels are sufficient as the mechanical system's primary responses are expected to be monotonically increasing or decreasing due to the geometrical variation within the limited solution space of $\pm 2.5 \%$ (see Table 1). Additionally, a center run is added to the design matrix to explore if any continuous variables' have second-order effects. The second-order effect will be confounded for all variables, i.e., it is unknown which variable is responsible for the non-linear behavior. However, if detected, the information passes to block 2 for further investigation (if the design concept passes to block 2). The chosen DOE configuration provides insight into the solution space's boundaries with limited confounding of possible two-factor interactions. Moreover, the DOE is suitable for augmentation in the subsequent block 2 (expanding the DOE with additional runs is an efficient method for reusing the data generated in block 1 ).
TABLE 2. OVERVIEW OF THE FEA SETUP AND CONDITIONS.

\begin{tabular}{l|l}
\hline Program & 3DX v. R2021x \\
Analysis type & Explicit \\
Controls & Mass scaling active \\
Component A & $\begin{array}{l}\text { Material properties elastic plastic, material } \\
\text { type plastic, half symmetry }\end{array}$ \\
Component B & $\begin{array}{l}\text { Material properties rigid, material type } \\
\text { plastic, section of ring }\end{array}$ \\
Mesh & $\begin{array}{l}\text { 3D quadratic, C3D10M, 54000 elements, } \\
\text { refinement contact and strain zones. } \\
\text { 200 elements for strain output, 200 ele- } \\
\text { ments for contact pressure output }\end{array}$ \\
Element & $\begin{array}{l}\text { Global contact, Coulomb friction of } 0.1 \\
\text { result groups } \\
\text { Contact }\end{array}$ \\
Restraints & $\begin{array}{l}\text { Fixed displacement control } \\
\text { Translation and rotation, applied through } \\
\text { connector nodes } \\
\text { Loads }\end{array}$ \\
Ansembly (0.001s), Rotation (0.008s)
\end{tabular}

\section{Block 1 - Design Review}

The purpose of the review is to evaluate whether a design concept shows reasonable performance for further optimization in block 2. No universal performance limits are provided as these are highly case-dependent. However, the following provides certain guidelines for the decision; i) Evaluate the significant variables. Effect sparsity is required for fractional factorial designs. ii) Evaluate the variable sensitivities. Avoid design concepts with high sensitivity within the range of EUV. iii) Evaluate the ratio of simulations in conflict with one or more constraints. Design concepts with a high ratio of feasible solutions indicate higher robustness.

\section{Block 2 - Augmented DOE}

Block 2 initiates with augmentation of the experimental design based on the screening design results from block 1 . The purpose of the augmentation is to create sufficient sampling points to generate meta-models suitable for optimization. The exploration focuses on the statistically significant variables determined in block 1 to minimize the required number of additional simulations. The augmentation can extend the explored solutions space or add additional points between the already explored bounds. In this case study, the augmentation focuses on adding axial and center points to produce a Central Composite Design, i.e., suitable for quadratic meta-models with interactions. The experimental design matrices are shown in appendix A and B. Other types of augmentation may be more appropriate if highly nonlinear and non-monotonic behavior is expected. A thorough 
overview of experimental designs is provided by Myers et al. [21], and Montgomery [22].

\section{Block 2 - Meta-model and Validation}

The meta-model aims to perform cost-efficient evaluations of the design concept for several variable combinations to estimate the probabilistic performance. The classical meta-model approaches are polynomial, neural network, and Kriging. However, depending on the DOE selection and the system response, the meta-model selection may vary. A methodology for fitting and validating meta-models is provided by Kleijnen et al. [23]. In the present case study, based on the Central Composite Design, polynomial meta-models are achieved using a least-square fit generated with SAS JMP [24]. However, adding this extra layer of abstraction can reduce accuracy. Therefore, to assess the meta-models' accuracy, it is recommended to perform additional FEAs, with new geometrical configurations within the SSV and compare the results. Thereby separating the metamodel training- and test data to measure the accuracy. In this case study, two extra FEAs were performed for this purpose. This case study did not follow an exact definition for the number of validation FEA. However, two were deemed sufficient for checking the accuracy and avoid overfitting. Depending on the complexity of the meta-model, more or fewer validation simulations could be required. The geometry dimensions were generated manually by selecting values in-between the DOE limits. Additionally, physical testing was performed on ten test-patch components to ensure the validity of the FEA.

\section{Block 2 - Optimization}

The multi-objective optimization problem formulation for probability of failure and standard deviation is shown in Eqs. (1) and (2) in negative null-form. The probability of failure is denoted $P$, and the standard deviation is $\sigma$. The meta-models for torque $\left(f_{\text {torque }}\right)$, strain $\left(f_{\text {strain }}\right)$, and contact pressure $\left(f_{\text {pressure }}\right)$ are presented in Eqs. (3), (4), and (5). The first optimization (Eq. (1)) explores the trade-off between probability of failure $(P)$ and standard deviation $(\sigma)$. The goal is to simultaneously identify design variables that improve both criteria with respect to the geometrical bounds and response constraints $\left(g_{j}\right)$. However, due to an unfavorable trade-off, the optimization was later chanced. So instead, Eq. (2) only focuses on the minimization of the failure rate.

$$
\begin{array}{cl}
\min _{x_{i}} & \left\{\max \left\{P_{j}\left(g_{j}\left(x_{i}\right)>0\right)\right\}, \max \left\{\sigma_{g_{j}}\left(x_{i}\right)\right\}\right\}, j=1 \ldots 3 \\
\text { s.t. } & g 1\left(x_{i}\right)=90 \mathrm{Nmm}-f_{\text {Torque }}\left(x_{i}\right) \leq 0 \\
& g 2\left(x_{i}\right)=f_{\text {Strain }}\left(x_{i}\right)-0.024 \leq 0 \\
& g 3\left(x_{i}\right)=f_{\text {Contact }}\left(x_{i}\right)-75 \mathrm{MPa} \leq 0 \\
& x_{i}-x_{i} S S V \leq x_{i} \leq x_{i}+x_{i} S S V
\end{array}
$$

$$
\begin{array}{ll}
\min _{x_{i}} & \sum_{j=1}^{3} P_{j}\left(g_{j}\left(x_{i}\right)>0\right) \\
\text { s.t. } & g 1\left(x_{i}\right)=90 \mathrm{Nmm}-f_{\text {Torque }}\left(x_{i}\right) \leq 0 \\
& g 2\left(x_{i}\right)=f_{\text {Strain }}\left(x_{i}\right)-0.024 \leq 0 \\
& g 3\left(x_{i}\right)=f_{\text {Contact }}\left(x_{i}\right)-75 \mathrm{MPa} \leq 0 \\
& x_{i}-x_{i} S S V \leq x_{i} \leq x_{i}+x_{i} S S V
\end{array}
$$

A Non-dominated Sorting Genetic Algorithm II (NSGA-II) is used to solve the multi-objective optimization problem [25]. The standard NSGA-II formulation is modified to include an inner Monte-Carlo simulation to generate 5000 normal distributed sampling points for each feasible solution to measure robustness and reliability. The measures of robustness and reliability are the standard deviation and the probability of failure of the distributions. In the original $R^{2} B D O$, the robustness and reliability measure is often defined as inequality constraints. However, in this case study, the measures are defined as the minimization objectives, and the functional responses are instead defined as inequality constraints. The switch enables visualization of the feasible solution space without a limit for the failure rate. By adopting this approach, the optimization can be performed with different EUV (representing geometrical tolerances), and the trade-off between tolerances and failure rates can be estimated. Finally, to increase the optimization result's confidence, an FEA with the optimized variables is performed to confirm the nominal performance.

\section{RESULTS}

The main results based on the new $s R^{2} B D O$ are provided in the following section. The main results are; i) Passing the design review in block 1 based on a sensitivity study and the solution space, ii) Augmenting the DOE and meta-models, iii) Robust and reliability optimization of the design concept, and iv) Evaluation of the new $s R^{2} B D O$ by comparing the optimization results with traditional $R^{2} B D O$ performed on the same geometry.

\section{Passing the Design Review}

Figure 6 shows the sensitivity result of the screening DOE. Six out of the twelve variables are statistically significant, with a confidence level of 0.05 . The size of the triangle indicates the magnitude of the variable sensitivity. As all the variables have upper and lower bounds of $\pm 2.5 \%$, the impact of variable variation is directly comparable. The comparison indicates that the radius, $R$, (see Fig. 5) has the highest impact on the design responses, meaning that variations in $R$ that occur in the production can cause high unwanted response variations. Figure 7 shows the torque versus time as the simulation progresses. Each curve represents a geometrical configuration. The minimum torque requirement is $90 \mathrm{Nmm}$ (indicated by the red hatch region). The 


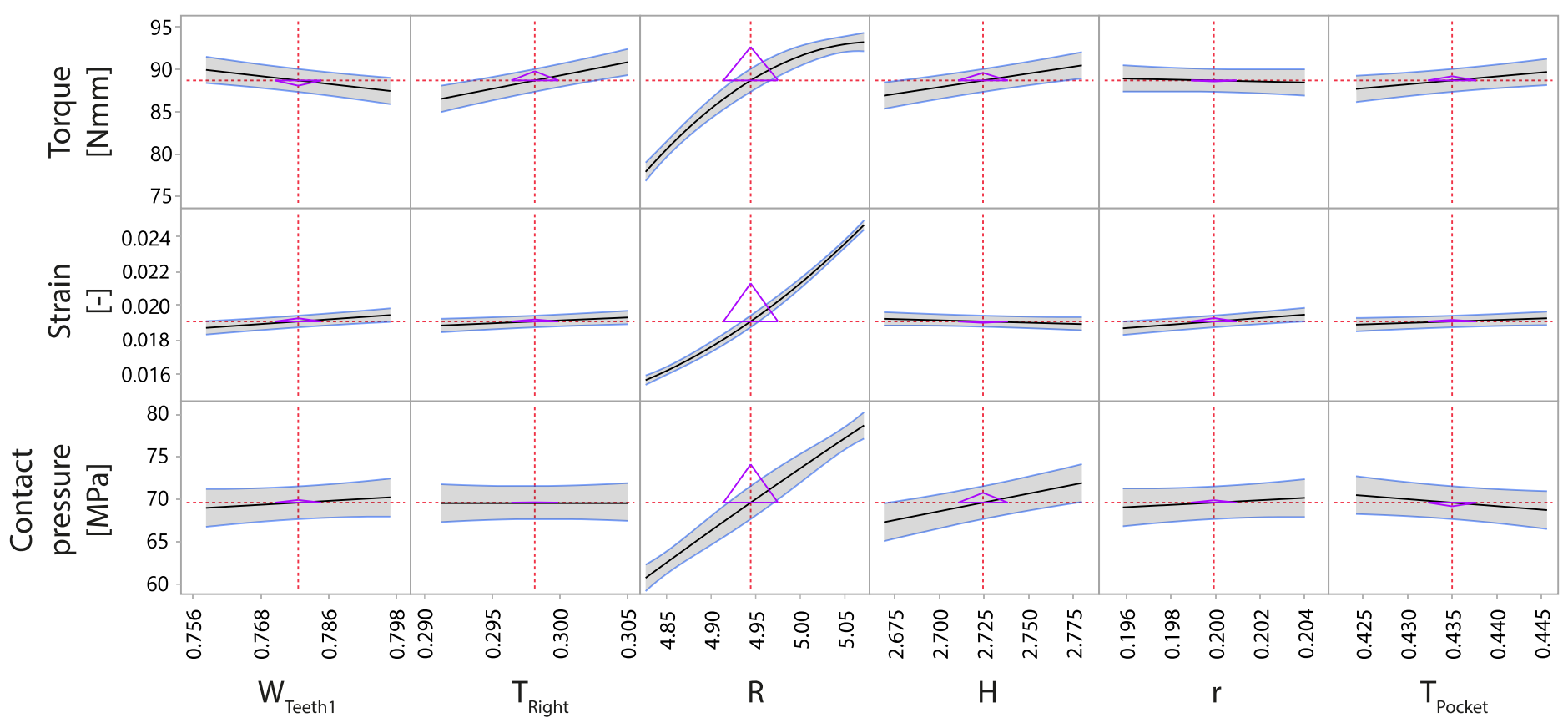

FIGURE 6. THE SENSITIVITY RESULT OF THE SCREENING DOE. SIX OUT OF THE TWELVE VARIABLE PROVED STATISTICALLY SIGNIFICANT.

purpose of the illustration is twofold: 1) Visualize the spread of the curves to indicate the robustness of the design. Despite a rather small solution space, a relatively large range between the curves points to low robustness. 2) Visualize the ratio between feasible and non-feasible solutions in the investigated solution space. In this case study, a quarter of the simulations provide a torque response above the lower limit. Similar graphs are created for the strain and contact pressure. The design concept passed the review as a sufficient portion (subjective and case dependent) of the simulations do not conflict with the three objective constraints. However, the design concept indicates low robustness as $\pm 2.5 \%$ input variation causes $28 \%$ output variation in the torque and conflicting boundary conditions.

\section{Augmentation and Meta-models}

The first step in block 2 is to augment the screening DOE (from block 1). With the suggested approach, adding axial and center points for the six significant variables, the augmentation only requires twelve additional simulations to achieve a Central Composite Design. Assuming monotonic responses, this results in second-order polynomial meta-models in only 45 simulations. While directly creating a Central Composite Design for twelve initial variables would require 284 simulations [26] (cementing a substantial gain by adopting the new $s R^{2} B D O$ framework). Figure 8 illustrates how the combined sampling points can create the three polynomial meta-models using a least-square fit. The meta-

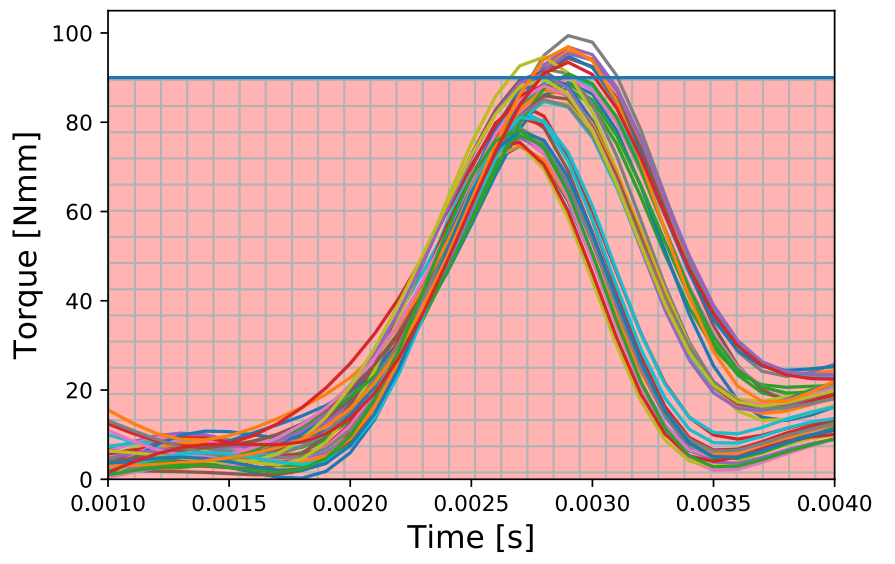

FIGURE 7. THE 33 INITIAL TORQUE CURVES WITH THE MINIMUM REQUIREMENT OF 90NMM (RED HATCH). VISUALIZING THE SPREAD IN PERFORMANCE AND FEASIBLE SOLUTION SPACE DUE TO GEOMETRICAL VARIATION OF $\pm 2.5 \%$.

models for the torque, strain, and contact pressure are shown in Eqs. (3), (4), and (5) with R-square values of 0.94, 0.98, and 0.91 , respectively. The meta-models responses were compared with the two extra FEAs with new geometrical combinations that were not used to fit the meta-model. The responses deviated by 


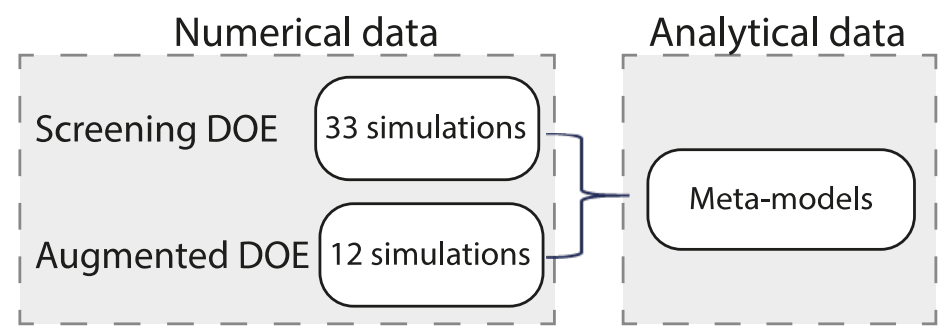

FIGURE 8. DATA FLOW FOR THE CREATION OF THE ANALYTICAL META-MODELS. THE NEW FRAMEWORK LIMITS THE NUMBER OF NEW SIMULATIONS REQUIRED TO CREATE ACCURATE META-MODELS FOR OPTIMIZATION IN BLOCK 2.

less than $1.5 \%$. Further validation of the FEA and meta-model accuracy were performed through physical torque testing on ten test-patch components. The components were manufactured using a single cavity molding form. Therefore, based on experience, the variation is expected to be less than the applied EUV; however, exact computer tomography was not performed. The nominal torque based on the meta-model is within $5 \%$ of the sample mean for the ten physical tests ( $s t d=7.5)$. Based on the physical testing and the FEA comparison, the meta-models were concluded to have an accuracy suitable for the subsequent optimization.

$$
\begin{gathered}
f_{\text {Torque }}\left(x_{i}\right)=-0.332-0.062 x_{1}+0.085 x_{3}+0.270 x_{4} \\
+0.058 x_{7}+0.025 x_{8}+\left(x_{3}-0.436\right) \\
\left(0.544\left(x_{7}-4.95\right)\right)-0.208\left(x_{7}-4.95\right)^{2} \\
f_{\text {Strain }}\left(x_{i}\right)=-0.212+0.021 x_{1}+0.018 x_{3}+0.032 x_{4} \\
+0.038 x_{7}-0.002 x_{8}+0.082+0.082 x_{12} \\
+0.137\left(x_{1}-0.778\right)\left(x_{7}-4.95\right)-0.478\left(x_{3}\right. \\
-0.436)\left(x_{8}-2.724\right)+0.063\left(x_{7}-4.95\right)^{2} \\
f_{\text {Contact }}\left(x_{i}\right)=-319.2 e 6-74.8 e 6 x_{3}+67.6 e 6 x_{7}+31.7 e 6 x_{8} \\
+446.2 e 6\left(x_{1}-0.778\right)\left(x_{7}-4.95\right)-1043.9 e 6 \\
\left(x_{3}-0.436\right)\left(x_{8}-2.724\right)-105.9 e 6\left(x_{7}-4.95\right) \\
\left(x_{8}-2.724\right)-1442.9 e 6\left(x_{7}-4.95\right)\left(x_{12}-0.2\right)
\end{gathered}
$$

The $x_{i}$ vector is tied to the model parameters in Table 4 .

\section{Optimization Exploration}

The results from the first optimization (Eq. (1)) is shown in 9. The figure shows the Pareto front between the probability of failure $(P)$ and the normalized standard deviation $(\sigma)$. Ideally, a

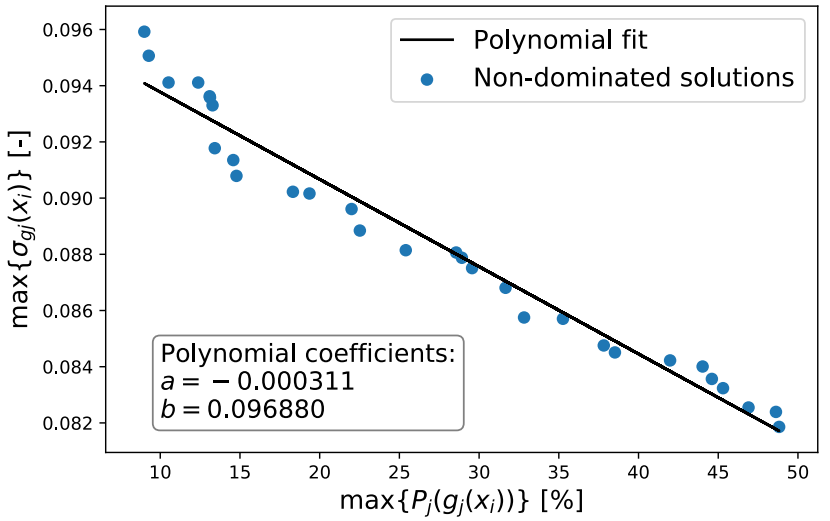

FIGURE 9. PARETO FRONT OF THE NON-DOMINATED SOLUTIONS BETWEEN THE MAXIMUM FAILURE RATES AND THE MAXIMUM STANDARD DEVIATION (NORMALIZED). A LINEAR POLYNOMIAL FIT IS ADDED TO SHOW THE TRADEOFF VALUES, I.E., FOR A 1\% INCREASE OF FAILURE RATE, A REDUCTION OF ONLY 0.000311 STANDARD DEVIATIONS IS ACHIEVED. AS A RESULT OF THIS TRADE-OFF, THE FAILURE RATE IS DEEMED THE DOMINATING OBJECTIVE TO MINIMIZE.

positive relationship is identified as it would allow reliability and robustness to improve simultaneously. Unfortunately, it is not achieved in this case study. Instead, a conflicting linear tradeoff exists between the failure rate $(P)$ and the standard deviation (sigma). The trade-off is not in favor of standard deviation as the failure rate increases dramatically with only marginal gain in robustness, i.e., a reduction in standard deviation.

As a consequence optimization objective was changed to minimize and explore the individual failures, see Eq. (2). Figure 10 shows the Pareto front of the individual failure rates. The optimized design is located with the smallest distances to the utopia point. Therefore, it will have the lowest combined failure rate as all three failure modes are considered equally important. Figure 11 shows the results generated by the optimization scheme. The nominal performance of the optimized design (blue), the probabilistic performance distribution of the optimized design (green), and the feasible domain (grey). The grey points are all the feasible design solutions, bounded by a $\pm 2.5 \%$ geometrical SSV, constrained by the functional requirements (red hatched area). The probabilistic performance is based on the EUV $\pm 1.0 \%$. The black dot represents the original design performance. The original nominal design performs outside the constraints and results in a failure rate of $78 \%$. The optimized design (blue) obtains a minimum failure rate of $8.6 \%$, see Table 3. However, even with such a substantial improvement in the failure 


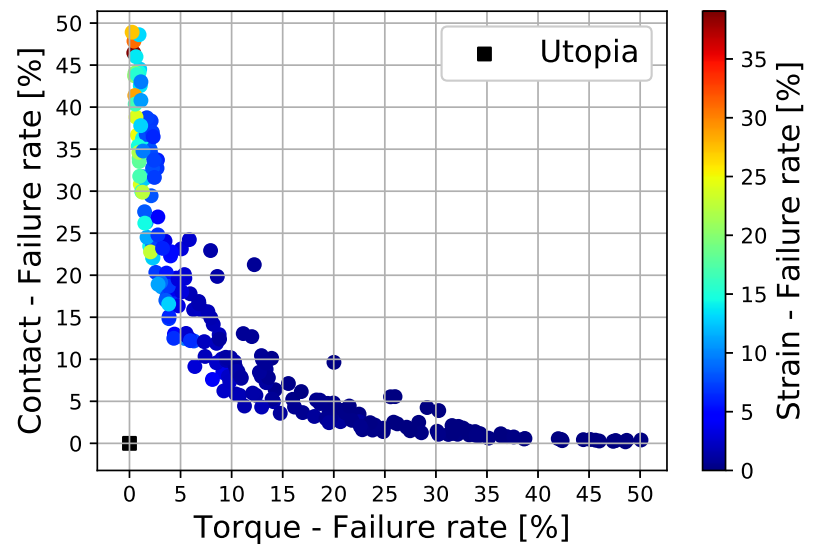

FIGURE 10. PARETO FRONT OF NON-DOMINATED SOLUTIONS FOR THE SEPARATE FAILURE RATES.

rate, it remains outside the allowable limit for large-scale plastic molding production. The result leaves two options; i) test failure rates with a lower EUV (tightening of tolerances), or ii) redesign the design concept in block 1 based on the new knowledge of sensitive variables.

In Fig. 12, the optimization is repeated with the EUV reduced to $\pm 0.5 \%$. The optimization generates the same geometrical variable values as before but reduces the maximum failure rate to $0.2 \%$, suitable for large-scale production. However, tightening the tolerances typically increases the cost of production, and it should be evaluated if the design concept remains cost-efficient. A summary of the reliability and robustness performance is shown in Table 3. Each design is evaluated based on two levels of EUV; \pm 0.5 and $\pm 1 \%$. The first column section shows the performance of the original design. The second column sections show the performance of the optimized design. The first row section shows the nominal performance of torque, strain, and contact pressure. The middle row section shows the robustness scores in the form of the normalized standard deviations. Finally, the bottom row section shows the failure rates. The original design performance did not meet the torque requirement, which should be above $90 \mathrm{Nmm}$. It was also noted in Table 3 that the failure rate of the original design concept increases with a reduced EUV. Initially, this might seem counter-intuitive. However, the reason is identified in the shape of the probability distributions, see green in Figs. 11 and 12. The input variables follow a normal distribution, while the output of the torque metamodel is Gamma/Poisson shaped. Due to the nominal performance, and the shape of the Monte-Carlo distribution, more design samples fall below the torque requirement with lower EUV. A surprising case where tightening of the tolerances would result in a worse-performing design when considering variation. It was concluded that the observed minimal difference in nominal
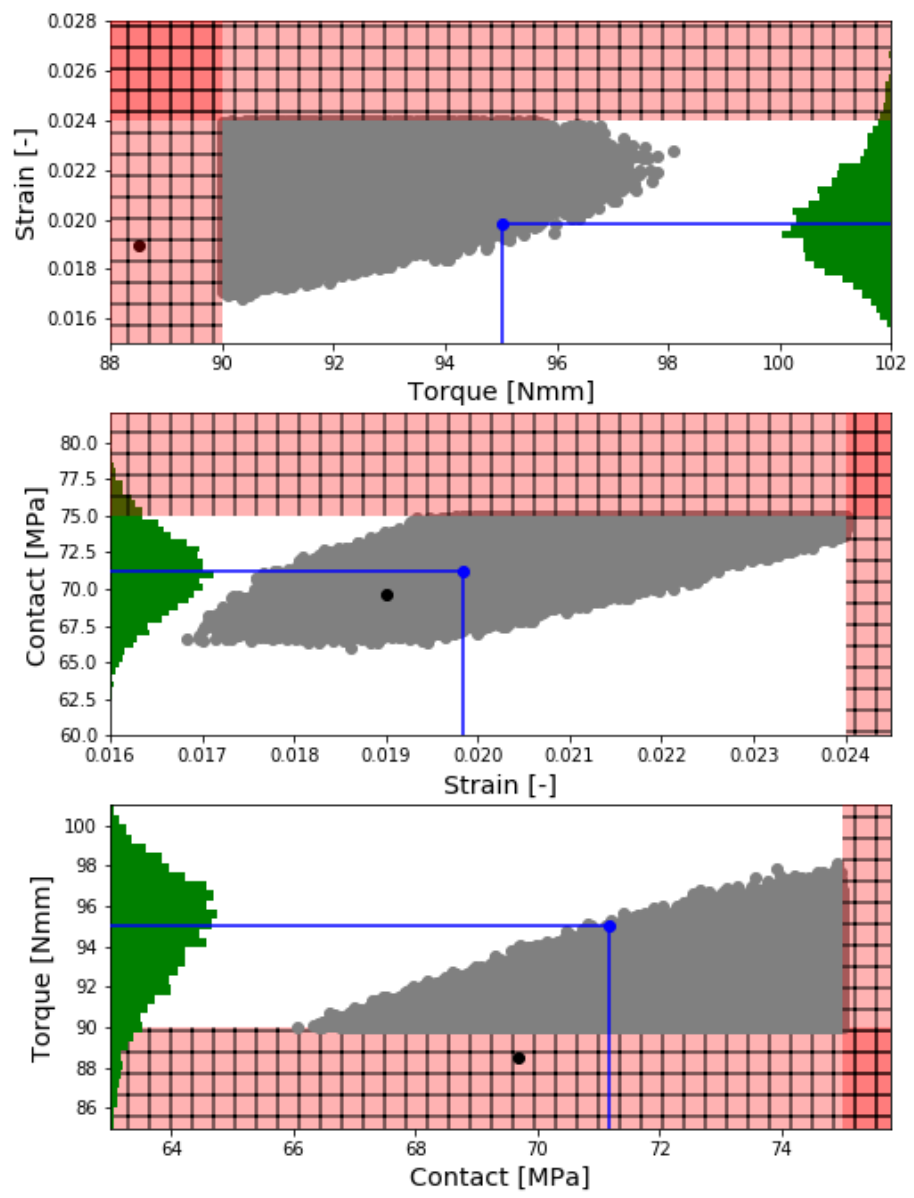

FIGURE 11. VISUALIZATION OF THE SOLUTION SPACE WITH $\pm 1 \%$ EUV OF THE RELIABILITY-BASED OPTIMIZATION PROBLEM. ORIGINAL DESIGN (BLACK). OPTIMIZED DESIGN (BLUE).

performance is related to the stochastic noise from the MonteCarlo simulations. Table 4 shows a comparison of the geometrical variables between the original- and the optimized design. The optimization results are pushing $W_{\text {Teeth } 1}, T_{\text {Pocket }}$, and $r$ to their boundary values of the solution space (maximum of $\pm 2.5 \%$ ). The boundary case indicates that further optimization could be possible with an enlarged solution space. Evaluation of these boundary cases obtains critical information, mainly when the optimization results in no feasible solutions. The remaining three variables are within the investigated solution space. Further expansion of these variables ranges is less likely to increase performance (unless highly non-linear effects are present). The final optimized geometry was checked by re-running the FEA. The FEA outputs are; torque $=93.8 \mathrm{Nmm}$, strain $=0.0193$, and contact pressure $=70.5 \mathrm{MPa}$. The outputs are marginally lower compared to the optimized meta-model results in Table 3. 

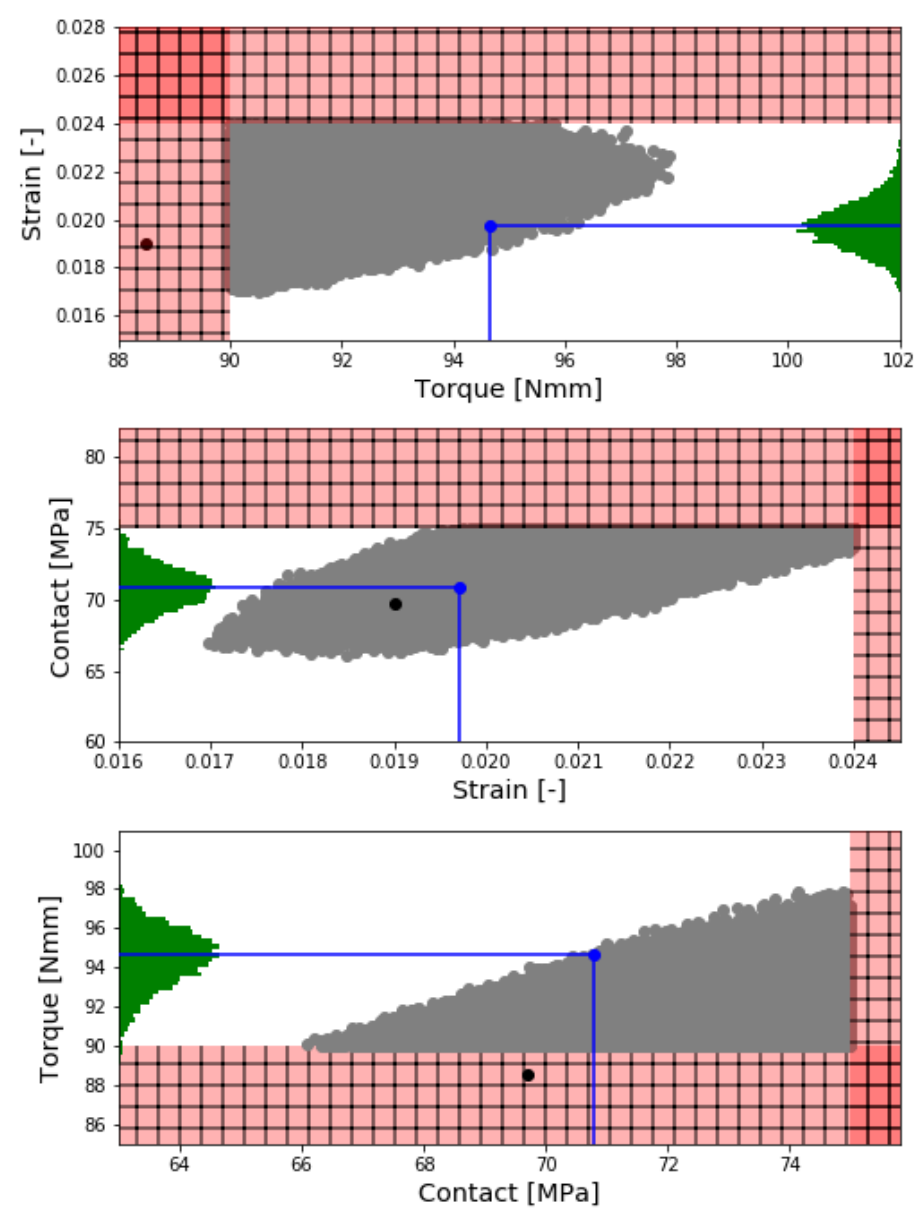

FIGURE 12. VISUALIZATION OF THE SOLUTION SPACE WITH $\pm 0.5 \%$ EUV OF THE RELIABILITY-BASED OPTIMIZATION PROBLEM. ORIGINAL DESIGN (BLACK). OPTIMIZED DESIGN (BLUE).

\section{Evaluation of the New Framework}

Finally, to indicate whether the suggested framework is efficient while maintaining accuracy, a direct comparison is made between the meta-model based on blocks 1 and 2 and a metamodel based on the original $R^{2} B D O$, using the additional 120 simulations based on an Optimal Latin Hypercube design. The overview of comparable results is shown in Table 5. All results are based on the same optimized geometrical dimensions, as seen in Table 4. The overview shows the meta-model based on the original $R^{2} B D O$ underestimates the torque compared to the FEA by $2.24 \%$. In contrast, the meta-model based on the new $s R^{2} B D O$ overestimates the torque by $1.17 \%$. In this case, the new $s R^{2} B D O$ framework achieves similar optimization results as the original $R^{2} B D O$ while minimizing the required simulations, a reduction of $62.5 \%$ (from 120 to only 45 simulations).
TABLE 3. PERFORMANCE COMPARISON BETWEEN THE ORIGINAL AND OPTIMIZED DESIGN. $\sigma$ : NORMALIZED STANDARD DEVIATION. $P$ : PROBABILITY OF FAILURE.

\begin{tabular}{l|ll|ll|l}
\hline & \multicolumn{2}{c}{ Original } & \multicolumn{2}{c}{ Optimized } \\
\multicolumn{1}{c}{ performance } & \multicolumn{2}{c}{ performance } \\
\hline EUV & \pm 1 & \pm 0.5 & \pm 1 & \pm 0.5 & $\%$ \\
\hline Torque & 88.4 & 88.7 & 94.9 & 94.8 & Nmm \\
Strain & 0.019 & 0.019 & 0.020 & 0.020 & - \\
Contact & 69.7 & 69.6 & 70.9 & 70.9 & $\mathrm{MPa}$ \\
\hline$\sigma_{\text {Torque }}$ & 0.036 & 0.018 & 0.033 & 0.015 & - \\
$\sigma_{\text {Strain }}$ & 0.098 & 0.049 & 0.095 & 0.047 & - \\
$\sigma_{\text {Contact }}$ & 0.055 & 0.028 & 0.043 & 0.021 & - \\
\hline$P_{\text {Torque }}$ & 66.6 & 78.0 & 8.6 & 0.2 & $\%$ \\
$P_{\text {Strain }}$ & 2.0 & 0.0 & 2.6 & 0.1 & $\%$ \\
$P_{\text {Contact }}$ & 7.9 & 0.2 & 8.2 & 0.2 & $\%$ \\
\hline
\end{tabular}

TABLE 4. A COMPARISON OF THE GEOMETRICAL VARIABLES BETWEEN THE ORIGINAL AND THE OPTIMIZED DESIGN.

\begin{tabular}{|c|c|c|c|c|}
\hline Variable & Original & Optimized & Var. & Var. \\
\hline Name/Sym. & Design & Design & Unit & Change \\
\hline$W_{\text {Teeth } 1} / x_{1}$ & 0.778 & 0.759 & $\mathrm{~mm}$ & $-2.44 \%$ \\
\hline$T_{\text {Pocket }} / x_{3}$ & 0.436 & 0.447 & $\mathrm{~mm}$ & $2.50 \%$ \\
\hline$T_{\text {Right }} / x_{4}$ & 0.300 & 0.306 & $\mathrm{~mm}$ & $2.00 \%$ \\
\hline $\mathrm{R} / x_{7}$ & 4.950 & 4.980 & $\mathrm{~mm}$ & $0.61 \%$ \\
\hline $\mathrm{H} / x_{8}$ & 2.724 & 2.741 & $\mathrm{~mm}$ & $0.62 \%$ \\
\hline $\mathrm{r} / x_{12}$ & 0.195 & 0.200 & $\mathrm{~mm}$ & $2.44 \%$ \\
\hline
\end{tabular}

\section{Discussion}

The study results indicate that the new $s R^{2} B D O$ framework is suitable for screening feasible design concepts early in the embodiment phase and subsequently optimize their probabilistic performance. In this study, the new framework reduces the computational effort significantly $(62.5 \%)$ while maintaining accuracy, increasing the possible use of FEA-based variation simulation in the early embodiment phase. Consequently, design issues can be detected earlier, which potentially can reduce the costs related to late-stage design changes. Instead, the engineering effort can be spent on further optimization and improving 
TABLE 5. A COMPARISON OF OPTIMIZATION RESULTS BETWEEN THE NEW $s R^{2} B D O$ AND THE ORIGINAL $R^{2} B D O$.

\begin{tabular}{r|c|c|}
\multicolumn{1}{c}{} & \multicolumn{1}{c}{ Torque $[\mathrm{Nmm}]$} & Diviation from FEA [\%] \\
\cline { 2 - 3 } FEA & 93.8 & - \\
\cline { 2 - 3 } New $s R^{2} B D O$ & 94.9 & +1.17 \\
\cline { 2 - 3 } Original $R^{2} B D O$ & 91.7 & -2.24 \\
\cline { 2 - 3 } & &
\end{tabular}

customer satisfaction and safety by producing the most robust and reliable product. While the prospects of the new framework are attractive, some limitations will require further exploration and research. (1) The reduction in computation effort will be case-dependent and correlate with response type and the required accuracy of the meta-model. In this case, the meta-models for the functional requirements were linear and quadratic. However, if the meta-models require higher-order polynomials to represent the structural behavior, a more extensive DOE augmentation is expected in block 2, resulting in higher computation needs. (2) Performing probabilistic evaluations in the tolerance range is challenged by the accumulative error. In this case study, the accuracy of the meta-model and the EUV are within the range of a few percent. When possible, it is recommended to have an order of magnitude larger EUV than the meta-model error to separate the probabilistic performance and the noise. Therefore, a future research study is suggested to compare a known performance distribution from physical production and the virtually estimated probabilistic performance. By knowing the true distribution of the production sample, the framework could be further validated. It would also allow a better foundation for testing different combinations of DOEs and meta-models, generating the most accurate results.

\section{CONCLUSION}

The key contribution is a new $s R^{2} B D O$ framework presented in Fig. 1, suitable for mechanical development of design concepts with limited legacy knowledge and with simulation resources available. By separating design insight (block 1) and design optimization (block 2), computational resources are used more efficiently while maintaining high accuracy. Moreover, the $s R^{2} B D O$ framework aligns with the typical design process seen in many industries. The $s R^{2} B D O$ framework allows for the initial efficient screening of multiple design concepts and only attempts optimization on design concepts with a promising feasible solution space.

The new $s R^{2} B D O$ framework is demonstrated on plastic molded components for a medical injection device. Block 1 uses a resolution-IV fractional factorial design with an added centerrun to screen the twelve included geometrical variables. Six variables showed as statistically significant and with the presence of a non-linear effect. The design review in block 1 concluded the design concept as sensitive towards geometrical variation but with a feasible solution space for optimization. In the subsequent block 2, an optimized design concept is achieved. The failure rate is reduced from $78 \%$ to $0.2 \%$ by adjusting the nominal values for the six variables and tightening the tolerances (EUV). The optimization was performed with meta-models based on an augmented DOE, which in the new $s R^{2} B D O$ is only required for the design concept deemed feasible. The meta-models were validated with additional FEA and physical experiments. The study finds that the new $s R^{2} B D O$ framework achieves high accuracy with a significant reduction in required simulations compared to the original $R^{2} B D O$ using Latin Hypercube Sampling.

\section{ACKNOWLEDGMENT}

The work is supported partly by Novo Nordisk A/S and partly by Innovation Fund Denmark, grant no.: 8053-00198A.

\section{REFERENCES}

[1] Doorn, N., and Hansson, S. O., 2011. "Should probabilistic design replace safety factors?". Philosophy and Technology, 24(2), pp. 151-168.

[2] Frangopol, D. M., and Maute, K., 2003. "Life-cycle reliability-based optimization of civil and aerospace structures". Computers and Structures, 81(7), pp. 397-410.

[3] Agarwal, H., Renaud, J. E., Lee, J. C., and Watson, L. T., 2004. "A unilevel method for reliability based design optimization". Collection of Technical Papers Aiaa/asmelascelahs/asc Structures, Structural Dynamics and Materials Conference, 7, pp. 5374-5392.

[4] Lee, K. H., and Park, G. J., 2001. "Robust optimization considering tolerances of design variables". Computers and Structures, 79(1), pp. 77-86.

[5] Messac, A., and Ismail-Yahaya, A., 2002. "Multiobjective robust design using physical programming". Structural and Multidisciplinary Optimization, 23(5), pp. 357-371.

[6] Beyer, H.-g., and Sendhoff, B., 2007. "Robust optimization - A comprehensive survey". Computer Methods in Applied Mechanics and Engineering, 196(33-34), pp. 3190-3218.

[7] Ju, B. H., and Lee, B. C., 2008. "Reliability-based design optimization using a moment method and a kriging metamodel". Engineering Optimization, 40(5), pp. 421-438.

[8] Lagaros, N. D., and Papadrakakis, M., 2007. "Robust seismic design optimization of steel structures". Structural and Multidisciplinary Optimization, 33(6), pp. 457-469.

[9] Lee, I., Choi, K. K., Du, L., and Gorsich, D., 2008. "Dimension reduction method for reliability-based robust design optimization". Computers and Structures, 86(13-14), pp. 1550-1562.

[10] Yadav, O. P., Bhamare, S. S., and Rathore, A., 2010. 
"Reliability-based robust design optimization: A multiobjective framework using hybrid quality loss function". Quality and Reliability Engineering International, 26(1), pp. 27-41.

[11] Paiva, R. M., Crawford, C., and Suleman, A., 2014. "Robust and reliability-based design optimization framework for wing design". AIAA Journal, 52(4), pp. 711-724.

[12] Sobek II, D. K., Ward, A. C., and Liker, J. K., 1999. "Toyota's principles of set-based concurrent engineering". MIT Sloan Management Review, 40(2), p. 67.

[13] Beitz, P. G., Feldhusen, W., and K.H., J. G., 2007. Engineering Design, third ed. Springer.

[14] Pohl, J., Thompson, H. M., Schlaps, R. C., Shahpar, S., Fico, V., and Clayton, G. A., 2017. "Innovative turbine stator well design using a kriging-assisted optimization method". Journal of Engineering for Gas Turbines and Power, 139(7), pp. 1-9.

[15] Du, X., Sudjianto, A., and Chen, W., 2004. “An integrated framework for optimization under uncertainty using inverse reliability strategy". Journal of Mechanical Design, Transactions of the ASME, 126(4), pp. 562-570.

[16] Iso.org, 2010. Geometrical product specifications (GPS) - ISO code system for tolerances on linear sizes - Part 1: Basis of tolerances, deviations and fits. ISO.

[17] Madrid, J., Lorin, S., Söderberg, R., Hammersberg, P., Wärmefjord, K., and Lööf, J., 2019. "A virtual design of experiments method to evaluate the effect of design andwelding parameters on weld quality in aerospace applications". Aerospace, 6(6).

[18] Gebhard R., 2013. 'A resilient modeling strategy' - Technical presentation. Tech. rep., Solid Edge University.

[19] Camba, J. D., Contero, M., and Company, P., 2016. "Parametric CAD modeling: An analysis of strategies for design reusability". CAD Computer Aided Design, 74, pp. 18-31.

[20] Hamada, M., Wu, C.-F., and Jeff, W. C.-F., 2000. Experiments : planning, analysis, and parameter design optimization. Wiley.

[21] Myers, R. H., and Montgomery, D. C., 2002. Response surface methodology : process and product optimization using designed experiments. Wiley.

[22] Montgomery, D. C., 2012. Design and Analysis of Experiments, Vol. 3. John Wiley \& Sons,.

[23] Kleijnen, J. P., and Sargent, R. G., 2000. “A methodology for fitting and validating metamodels in simulation". European Journal of Operational Research, 120(1), pp. 14-29.

[24] JMP, 2018. JMP 14 Deisng of Experiments Guide. SAS Institute.

[25] Deb, K., Pratap, A., Agarwal, S., and Meyarivan, T., 2002. "A fast and elitist multiobjective genetic algorithm: NSGAII". IEEE Transactions on Evolutionary Computation, 6(2), pp. 182-197.

[26] Sanchez, S. M., and Sanchez, P. J., 2005. "Very large fractional factorial and central composite designs". ACM Transactions on Modeling and Computer Simulation, 15(4), pp. 362-377. 


\section{Appendix A: DOE screening table}

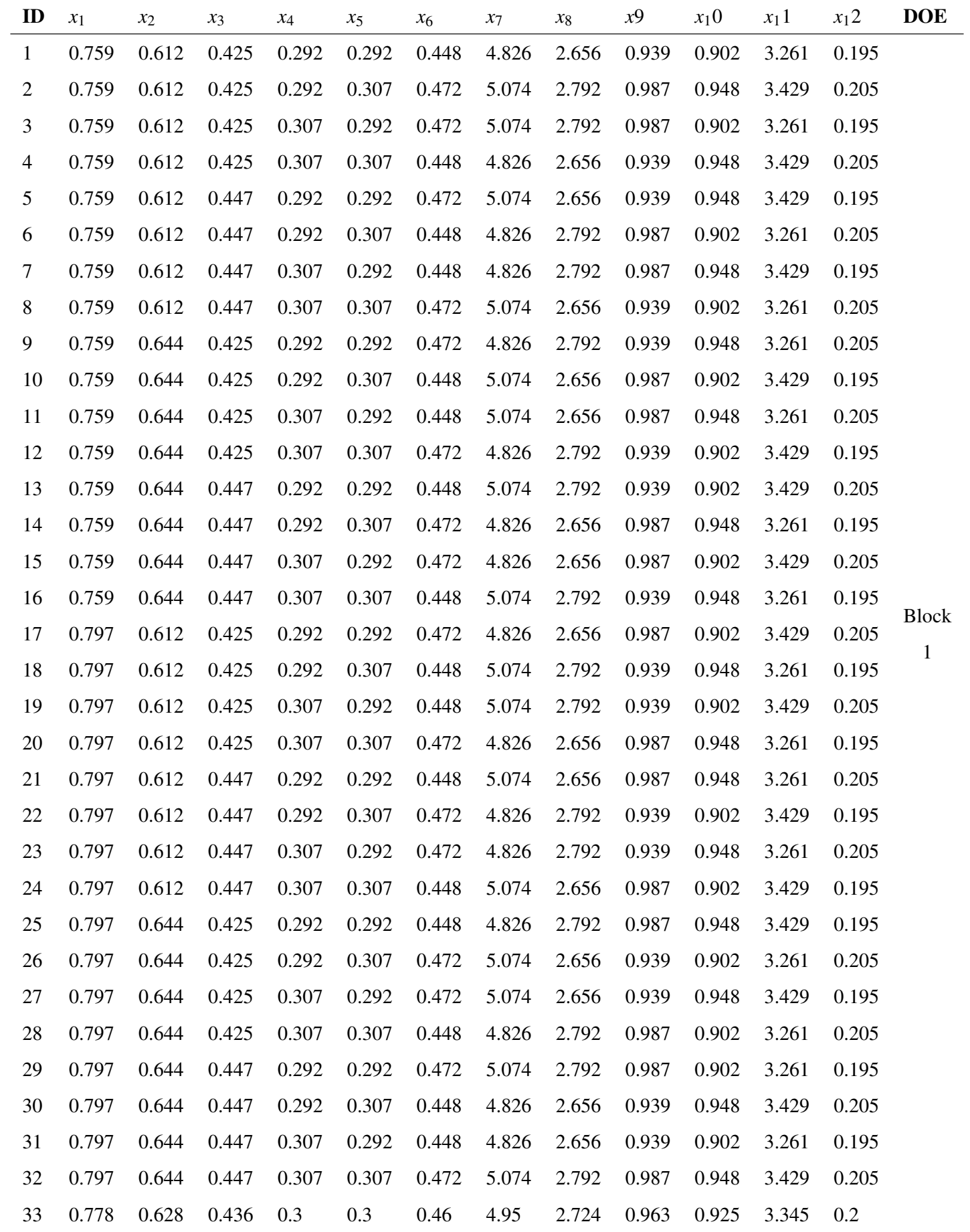


Appendix B: DOE augmentation and meta-model

check

\begin{tabular}{llllllllllllll} 
ID & $x_{1}$ & $x_{2}$ & $x_{3}$ & $x_{4}$ & $x_{5}$ & $x_{6}$ & $x_{7}$ & $x_{8}$ & $x 9$ & $x_{1} 0$ & $x_{1} 1$ & $x_{1} 2$ & DOE \\
\hline 34 & 0.778 & 0.628 & 0.436 & 0.3 & 0.2995 & 0.46 & 5.074 & 2.724 & 0.963 & 0.925 & 3.345 & 0.2 & \\
35 & 0.778 & 0.628 & 0.436 & 0.3 & 0.2995 & 0.46 & 4.826 & 2.724 & 0.963 & 0.925 & 3.345 & 0.2 & \\
36 & 0.778 & 0.628 & 0.436 & 0.3 & 0.307 & 0.46 & 4.95 & 2.724 & 0.963 & 0.925 & 3.345 & 0.2 & \\
37 & 0.778 & 0.628 & 0.436 & 0.3 & 0.292 & 0.46 & 4.95 & 2.724 & 0.963 & 0.925 & 3.345 & 0.2 & \\
38 & 0.778 & 0.628 & 0.436 & 0.3 & 0.2995 & 0.46 & 4.95 & 2.792 & 0.963 & 0.925 & 3.345 & 0.2 & \\
39 & 0.778 & 0.628 & 0.436 & 0.3 & 0.2995 & 0.46 & 4.95 & 2.656 & 0.963 & 0.925 & 3.345 & 0.2 & Block \\
40 & 0.797 & 0.628 & 0.436 & 0.3 & 0.2995 & 0.46 & 4.95 & 2.724 & 0.963 & 0.925 & 3.345 & 0.2 & 2 \\
41 & 0.759 & 0.628 & 0.436 & 0.3 & 0.2995 & 0.46 & 4.95 & 2.724 & 0.963 & 0.925 & 3.345 & 0.2 & \\
42 & 0.778 & 0.628 & 0.447 & 0.3 & 0.2995 & 0.46 & 4.95 & 2.724 & 0.963 & 0.925 & 3.345 & 0.2 & \\
43 & 0.778 & 0.628 & 0.425 & 0.3 & 0.2995 & 0.46 & 4.95 & 2.724 & 0.963 & 0.925 & 3.345 & 0.2 & model \\
44 & 0.778 & 0.628 & 0.436 & 0.3 & 0.2995 & 0.46 & 4.95 & 2.724 & 0.963 & 0.925 & 3.345 & 0.205 & check \\
45 & 0.778 & 0.628 & 0.436 & 0.3 & 0.2995 & 0.46 & 4.95 & 2.724 & 0.963 & 0.925 & 3.345 & 0.195 & \\
\hline 46 & 0.767 & 0.628 & 0.447 & 0.300 & 0.307 & 0.460 & 5.035 & 2.771 & 0.963 & 0.925 & 3.345 & 0.199 & Meta \\
47 & 0.769 & 0.628 & 0.439 & 0.300 & 0.312 & 0.460 & 4.964 & 2.773 & 0.963 & 0.925 & 3.345 & 0.197 & manch
\end{tabular}

